\title{
AN EQUICONVERGENCE THEOREM FOR A CLASS OF EIGENFUNCTION EXPANSIONS(')
}

\author{
BY \\ C. G. C. PITTS
}

\begin{abstract}
A recent result of Muckenhoupt concerning the convergence of the expansion of an arbitrary function in terms of the Hermite series of orthogonal polynomials is generalised to a class of orthogonal expansions which arise from an eigenfunction problem associated with a second-order linear differential equation.
\end{abstract}

1. Introduction. Szegö [6, p. 245, Theorem 9.1.6] has obtained two different sets of conditions which ensure the convergence of the expansion of an arbitrary function in terms of the orthogonal sequence of Hermite functions. The present author has generalized [4], [5] one of these results to a class of eigenfunction expansions which includes, as a special case, the Hermite expansion. Muckenhoupt [1, Theorem 2] has improved the convergence results of Szegö by relaxing the conditions that have to be satisfied by the arbitrary function; he has also shown [1, Theorem 4] that, in a particular sense, his hypotheses are the weakest possible. In the present paper it is shown how the methods of [4], [5] can be modified so as to obtain a result which includes as a special case the first of these results of Muckenhoupt.

The eigenfunction problem to be studied is defined by the differential equation

$$
y^{\prime \prime}(u)+\{\lambda-q(u)\} y(u)=0 \quad(0 \leq u<\infty)
$$

subject to the boundary condition

$$
y(0) \cos \alpha+y^{\prime}(0) \sin \alpha=0
$$

( $\alpha$ is a real constant) where $y(u)$ is to be $L^{2}(0, \infty)$. If $q(u)$ is continuous over $[0, \infty)$ and $q(u) \rightarrow \infty$ as $u \rightarrow \infty$, then it is well known that this problem possesses an increasing sequence $\lambda_{n}(n=0,1,2, \ldots)$ of eigenvalues with corresponding normalised eigenfunctions $\psi_{n}(u)$, say. We have an analogous situation if (1.1) is defined over $(-\infty, \infty)$ instead of $[0, \infty)$ and $y(u)$ is required to be $L^{2}(-\infty, \infty)$, where $q(u)$ is continuous over $(-\infty, \infty)$ and $q(u) \rightarrow \infty$ as $u \rightarrow \pm \infty$. The properties of the eigenfunction problem arising from the $(-\infty, \infty)$ case are exactly analogous to those of the $[0, \infty)$ case; it is therefore sufficient to discuss only the latter. We remark, however, that the convergence result which we shall

Received by the editors May 15, 1972 and, in revised form, December 10, 1972.

AMS (MOS) subject classifications (1970). Primary 34B25; Secondary 42 A60.

(1) The results of this paper were obtained while the author was on sabbatical leave at the University of British Columbia, Vancouver, Canada during the academic year 1971-1972. 
obtain, stated for the $(-\infty, \infty)$ case with $q(u)=u^{2}$, reduces exactly to the result of Muckenhoupt.

A full statement of the conditions on $q(u)$ that will be assumed is given in $\$ 2$ (there are two alternative sets of such conditions corresponding to the two situations considered in [4], [5]); the convergence result is then stated. In $\$ 3$ it is explained how the arguments of [2], [3], [4], [5] have to be modified so as to obtain the result of this paper. In $\$ \S 4,5$ some preliminary lemmas are established for the case in which $q(u)$ satisfies the first set of conditions; the proof of the main result is then given for these conditions in $\$ 6$. Lastly the variations necessary to cope with the alternative conditions on $q(u)$ are outlined in $\$ 7$.

No attempt has been made to establish analogues of Muckenhoupt's theorems concerning the best-possible nature of his results.

As usual $R, C$ denote the sets of real and complex numbers, $R^{+}=[0, \infty) ; A$, $B$ denote constants not necessarily the same at each appearance. The real and imaginary parts of the parameter $\lambda$ appearing in (1.1) are denoted by $\mu, \nu$.

Let $S$ be an unbounded set in $C$, and let $f, g$ be complex-valued functions defined on $S$. If there exist positive constants $A, B$ such that $A<|f(\lambda) / g(\lambda)|$ $<B$ for all $\lambda$ in $S$ with $|\lambda|$ sufficiently large, then we shall write $f(\lambda) \preceq g(\lambda)$ as $\lambda \rightarrow \infty$ in $S$.

Except where the contrary is stated we shall consider only the eigenvalue problem arising from the semi-infinite interval $[0, \infty)$.

2. The conditions on $q$; the results to be proved. Without loss of generality it is supposed that $q(0)=0$. It is then assumed that $q(u)$ satisfies one of the following sets of conditions.

The conditions $C_{\mathrm{e}}$. (i) The function $q(u)$ is real-valued, continuous, strictly increasing for all $u \geq 0$, and $q(u) \rightarrow \infty$ as $u \rightarrow \infty$.

(ii) Let $S_{\lambda}=\{\lambda$ : $|\arg \lambda|<\varepsilon, \lambda \in C\}$ where $\varepsilon$ is a positive constant. The function $q: R^{+} \rightarrow R^{+}$is the restriction of some complex-valued function defined on a set $S_{z}(\subseteq C)$ which possesses an inverse $p$ defined on $S_{\lambda}$ in such a way that $S_{z}=p\left(S_{\lambda}\right)$. This extension of $q: R^{+} \rightarrow R^{+}$will also be denoted by $q$; thus $S_{\lambda}=q\left(S_{z}\right)$. The constant $\varepsilon$ is sufficiently small for the approximations of [2], [3] to hold.

(iii) $q^{\prime \prime}(z) / q^{\prime}(z)=O\left(q^{\prime}(z) / q(z)\right), q^{\prime \prime \prime}(z) / q^{\prime \prime}(z)=O\left(q^{\prime}(z) / q(z)\right)$ as $|z| \rightarrow \infty, z$ $\in S_{z}$.

(iv) For some constant $c$ in $\left(0, \frac{3}{2}\right), q^{\prime}(z)=O\left\{|q(z)|^{c}\right\}$ as $|z| \rightarrow \infty, z \in S_{z}$.

The conditions $C_{1}$. (i) The function $q(u)$ is real-valued, continuous, strictly increasing and concave for all $u \geq 0$, and $q(u) \rightarrow \infty$ as $u \rightarrow \infty$.

(ii) Let $S_{\lambda}=\left\{\lambda:|\nu|<\varepsilon p(\mu) / p^{\prime}(\mu), \lambda \in C\right\}$ where $\varepsilon$ is a positive constant and $p: R^{+} \rightarrow R^{+}$is the inverse of $q: R^{+} \rightarrow R^{+}$. The function $q: R^{+} \rightarrow R^{+}$is the restriction of some complex-valued function defined on a set $S_{z}(\subseteq C)$ which possesses an inverse $p$ defined in such a way that $S_{z}=p\left(S_{\lambda}\right)$. This extension of $q: R^{+} \rightarrow R^{+}$will also be denoted by $q$; thus $S_{\lambda}=q\left(S_{z}\right)$. The constant $\varepsilon$ is sufficiently small for the approximations of [5] to hold. 
(iii) $q^{\prime}(z) / q(z)=O(1 / z), q^{\prime \prime}(z) / q^{\prime}(z)=O(1 / z), q^{\prime \prime \prime}(z) / q^{\prime \prime}(z)=O(1 / z)$ as $|z|$ $\rightarrow \infty, z \in S_{z}$.

(iv) For some constant $\kappa$ in $(0,1), p^{\prime}(\mu) / p(\mu)=o\left(\left\{p^{\prime}(\kappa \mu)\right\}^{2 / 3}\right)$ as $\mu \rightarrow+\infty$.

(v) $p(\lambda) \asymp p(\mu)$ for any $\lambda(=\mu+i v)$ in $S_{\lambda}$.

The conditions $C_{\mathrm{e}}$ (considered in [2], [3], [4]) permit $q(u)$ to behave, as $u \rightarrow \infty$, like $u^{c}(c>0), \exp u, \exp \exp u$, etc., while the conditions $C_{1}$ (considered in [5]) permit $q(u)$ to behave, as $u \rightarrow \infty$, like $u^{c}(0<c \leq 1), \log u, \log \log u$, etc.

Let $\lambda_{n}(n=0,1, \ldots)$ be the eigenvalues of the problem defined in $\$ 1$ and let $\psi_{n}(u)$ be the corresponding normalised eigenfunctions. Let $f: R^{+} \rightarrow R$ be an arbitrary function and define (formally)

$$
c_{n}=\int_{0}^{\infty} f(u) \psi_{n}(u) d u ;
$$

then we shall be concerned with the convergence of the series

$$
\sum_{n=0}^{\infty} c_{n} \psi_{n}(x)
$$

The main result to be proved is as follows.

Theorem 2.1. Suppose that $q(u)$ satisfies the conditions $C_{\mathrm{e}}$ or $C_{1}$. If $f(u) /(1+u)$ is $L(0, \infty)$ and

$$
\int_{p(\Lambda / 2)}^{p(\Lambda)} \frac{|f(u)|}{u\left[\Lambda+\left\{p^{\prime}(\Lambda)\right\}^{-2 / 3}-q(u)\right]^{1 / 4}} d u=o\left(\Lambda^{-1 / 4}\right)
$$

as $\Lambda \rightarrow \infty$, then for any $x>0$ the series (2.2) is equiconvergent with the ordinary Fourier series expansion of $f(u)$ at $u=x$.

As explained above (see also $[4,87]$ ) this result can be reformulated to cover the case of eigenfunction expansions arising from the differential equation (1.1) defined over $(-\infty, \infty)$ instead of $[0, \infty)$. Let $C_{e}^{\prime}, C_{1}^{\prime}$ denote analogous conditions for $q(u)$ over $(-\infty, 0]$; then we have the following result for the expansion of an arbitrary function $f: R \rightarrow R$.

Theorem 2.2. Suppose that $q(u)$ satisfies the conditions $C_{\mathrm{e}}$ or $C_{1}$ and $C_{\mathrm{e}}^{\prime}$ or $C_{1}^{\prime}$ (not necessarily respectively). If $f(u) /(1+|u|)$ is $L(-\infty, \infty)$ and

$$
\int_{p(\Lambda / 2)}^{p(\Lambda)} \frac{|f(u)|+|f(-u)|}{u\left[\Lambda+\left\{p^{\prime}(\Lambda)\right\}^{-2 / 3}-q(u)\right]^{1 / 4}} d u=o\left(\Lambda^{-1 / 4}\right)
$$

as $\Lambda \rightarrow \infty$, then for any finite $x$ the eigenfunction expansion of $f(u)$ at $u=x$ is equiconvergent with the ordinary Fourier series expansion of $f(u)$ at $u=x$.

If we set $q(u)=u^{2}$ in the differential equation (1.1) defined over $(-\infty, \infty)$, then the normalised eigenfunctions are

$$
\exp \left(-\frac{1}{2} u^{2}\right) H_{n}(u) / 2^{n / 2}(n !)^{1 / 2} \pi^{1 / 4}
$$


$(n=0,1,2, \ldots)$ where $H_{n}(u)$ is the $n$th Hermite polynomial, and Theorem 2.2 reduces to Muckenhoupt's Theorem 2 of [1], bearing in mind that we have incorporated the weight function $\exp \left(-\frac{1}{2} u^{2}\right)$, appearing in [1] in the normalised eigenfunctions.

The following result will also be established for the $[0, \infty)$ case.

Theorem 2.3. Let $1 \leq r \leq \infty$. If $q(u)$ satisfies the conditions $C_{\mathrm{e}}$ and

$$
\begin{aligned}
\left(\int_{p(\Lambda)}^{p(2 \Lambda)}|f(u)|^{r} d u\right)^{1 / r} & =o\left[\Lambda^{-1 / 4} p(\Lambda)\left\{p^{\prime}(\Lambda)\right\}^{-1 / 2+1 /(3 r)}\right] & & \text { if } 1 \leq r<\frac{4}{3}, \\
& =o\left[p(\Lambda)\left\{\Lambda p^{\prime}(\Lambda) \log \Lambda\right\}^{-1 / 4}\right] & & \text { if } r=\frac{4}{3}, \\
& =o\left[p(\Lambda)\left\{\Lambda p^{\prime}(\Lambda)\right\}^{-1+1 / r}\right] & & \text { if } r>\frac{4}{3},
\end{aligned}
$$

as $\Lambda \rightarrow \infty$, or if $q(u)$ satisfies the conditions $C_{1}$ and

$$
\begin{aligned}
\left(\int_{p(\Lambda)}^{p(k \Lambda)}|f(u)|^{r} d u\right)^{1 / r} & =o\left[\Lambda^{-1 / 4} p(\Lambda)\left\{p^{\prime}(k \Lambda)\right\}^{-1 / 2+1 /(3 r)}\right] & & \text { if } 1 \leq r<\frac{4}{3}, \\
& =o\left[p(\Lambda)\left\{\Lambda p^{\prime}(k \Lambda) \log \Lambda\right\}^{-1 / 4}\right] & & \text { if } r=\frac{4}{3}, \\
& =o\left[p(\Lambda)\left\{\Lambda p^{\prime}(k \Lambda)\right\}^{-1+1 / r}\right] & & \text { if } r>\frac{4}{3},
\end{aligned}
$$

as $\Lambda \rightarrow \infty$, for some constant $k>1$, then (2.3) holds.

Observe that, setting $r=2$, in the relevant parts of (2.4), (2.5) we obtain respectively (1.7) of [4], (4.2) of [5], so Theorem 2.1 of the present paper is a generalisation of Theorem 1.1 of [4] and Theorem 4.1 of [5].

3. Method of proof of Theorem 2.1. Let $\theta(u, \lambda), \phi(u, \lambda)$ be the solutions of (1.1) such that

$$
\begin{array}{ll}
\theta(0, \lambda)=\cos \alpha, & \theta^{\prime}(0, \lambda)=\sin \alpha, \\
\phi(0, \lambda)=\sin \alpha, & \phi^{\prime}(0, \lambda)=-\cos \alpha .
\end{array}
$$

Since $q(u) \rightarrow \infty$ as $u \rightarrow \infty$ it follows that (1.1) is in the limit-point case so there exists a unique function $m(\lambda)$ which is analytic for $\operatorname{im} \lambda \neq 0$ and such that $\psi(u, \lambda)=\theta(u, \lambda)+m(\lambda) \phi(u, \lambda)$ is $L^{2}(0, \infty)$ for im $\lambda \neq 0$. Let

$$
\Phi(x, \lambda)=\psi(x, \lambda) \int_{0}^{x} \phi(u, \lambda) f(u) d u+\phi(x, \lambda) \int_{x}^{\infty} \psi(u, \lambda) f(u) d u
$$

Then, as in [4], [5], the hypothesis that $f(u) /(1+u)$ is $L(0, \infty)$ ensures that the function $\Phi(x, \lambda)$ and the generalised Fourier coefficients $c_{n}$ exist. Furthermore $\Phi(x, \lambda)$ is an analytic function of $\lambda$ except at the points $\lambda_{n}$ where it has simple 
poles with residues $c_{n} \psi_{n}(x)$. To prove Theorem 2.1 we integrate $\Phi(x, \lambda)$ around a large contour $Q_{\lambda}$ in the $\lambda$-plane and let $Q_{\lambda}$ expand to infinity so that

$$
\lim _{Q_{\lambda} \rightarrow \infty} \frac{1}{2 \pi i} \int_{Q_{\lambda}} \Phi(x, \lambda) d \lambda=\sum_{n=0}^{\infty} c_{n} \psi_{n}(x) .
$$

The contour $Q_{\lambda}$ is defined as follows. Let $s=\sqrt{ } \lambda$ (where $s$ is positive when $\lambda$ is positive) and $s=\sigma+i t ; Q_{\lambda}$ is symmetrical about the real $\lambda$-axis, and the upper half of the contour corresponds to $\sigma=N, 0 \leq t \leq N ; t=N, 0 \leq \sigma \leq N$ in the $s$-plane. Then $N$ is allowed to take an increasing sequence of values in such a way that $Q_{\lambda}$ expands to infinity through a sequence of distinct positions which avoid the poles of $\Phi(x, \lambda)$; how this is done is explained in $\$ 7$ of [3]. Thus in the following when we say that $\lambda \in Q_{\lambda}$ we shall mean that $\lambda$ lies on one of the discrete positions of the contour.

It is then shown that the left side of (3.1) has the same limit as the ordinary Fourier series expansion of $f(u)$ at $u=x$. The method by which this was done in [4] for the $C_{e}$ case contained an error; in [5] a corrected version of the method was given for the $C_{1}$ case. In this version the $u$-interval of integration in the definition of $\Phi$ is divided into seven subintervals $E_{i}(i=1,2, \ldots, 7)$. Then it is shown that given any $\eta(>0)$, the contribution to the left side of (3.1) of the second and third $u$ subintervals together give the same quantity as arises from the ordinary Fourier series of $f(x)$, to within $O(\eta)$, and all the other terms contribute $O(\eta)$.

The intervals $E_{i}$ are defined as follows. Let $\eta(>0)$ be arbitrary; let $\delta(>0)$ be chosen so that

$$
\int_{x-\delta}^{x}|f(u)| d u<\eta, \quad \int_{x}^{x+\delta}|f(u)| d u<\eta
$$

(this is possible since $f(u) /(1+u)$ is $L(0, \infty))$. Then in the $C_{1}$ case the subintervals $E_{i}$ are

$$
\begin{gathered}
(0, x-\delta), \quad(x-\delta, x), \quad(x, x+\delta), \quad(x+\delta, U), \quad\left(U, p\left(k^{\prime-1} N^{2}\right)\right), \\
\left(p\left(k^{\prime-1} N^{2}\right), p\left(k^{\prime} N^{2}\right)\right), \quad\left(p\left(k^{\prime} N^{2}\right), \infty\right),
\end{gathered}
$$

where $k^{\prime}=\sqrt{ } k$ and $k$ is the constant appearing in the hypotheses of Theorem 4.1 of [5]. In the $C_{e}$ case the corresponding subintervals $E_{i}$, for the analogous argument to that used in [5], would be

$$
\begin{gathered}
(0, x-\delta), \quad(x-\delta, x), \quad(x, x+\delta), \quad(x+\delta, U), \quad\left(U, p\left(\frac{1}{2} N^{2}\right)\right), \\
\left(p\left(\frac{1}{2} N^{2}\right), p\left(4 N^{2}\right)\right), \quad\left(p\left(4 N^{2}\right), \infty\right) .
\end{gathered}
$$

With this definition of the intervals $E_{i}$ it is straightforward to give a valid proof of Theorem 1.1 of [4], using analogous arguments to those of [5]. 
It is clear that in the $C_{1}$ case all the arguments and conclusions of $\S \S 4,6$ of [5], which depend only on the hypothesis that $f(u) /(1+u)$ is $L(0, \infty)$, continue to hold under the hypotheses of Theorem 2.1 of the present paper. Thus in particular the arguments concerning the intervals $E_{i}(i=1,2,3,4,5,7)$ all hold, so the only part of the argument which requires a fresh appraisal is the contribution arising from $E_{6}$. In the same way it is also true that in the $C_{e}$ case we need only now consider $E_{6}$. Thus to prove Theorem 2.1 it is sufficient to prove that, as $N \rightarrow \infty$,

$$
\int_{Q_{\lambda}} \phi(x, \lambda)\left\{\int_{E_{6}} \psi(u, \lambda) f(u) d u\right\} d \lambda \rightarrow 0
$$

where $E_{6}=\left(p\left(\frac{1}{2} N^{2}\right), p\left(4 N^{2}\right)\right)$ in the $C_{\mathrm{e}}$ case and $E_{6}=\left(p\left(k^{\prime-1} N^{2}\right), p\left(k^{\prime} N^{2}\right)\right)$ in the $C_{1}$ case.

The difference in the definition of the intervals $E_{i}$ in the $C_{\mathrm{e}}$ and $C_{1}$ cases arises from the slightly different form of the hypotheses of the equiconvergence theorems in [4] and [5]. In the present paper the hypotheses are now identical in the two cases; thus it is more natural and also more convenient to define the intervals $E_{i}$ in the same way in the two cases. We therefore define them by (3.3) in both cases. In fact the treatment of the intervals $E_{5}, E_{7}$ that was used in $\$ 6$ of [5] is not essentially affected by such a change. Hence Theorem 2.1 will be proved if we can show that, as $N \rightarrow \infty$,

$$
\int_{Q_{\lambda}} \phi(x, \lambda)\left\{\int_{p\left(N^{2} / 2\right)}^{p\left(4 N^{2}\right)} \psi(u, \lambda) f(u) d u\right\} d \lambda \rightarrow 0 .
$$

We make one further remark. It can easily be shown that the hypothesis (2.3) is equivalent to the requirement that

$$
\int_{p(k \Lambda)}^{p(\Lambda)} \frac{|f(u)|}{u\left[\Lambda+\left\{p^{\prime}(\Lambda)\right\}^{-2 / 3}-q(u)\right]^{1 / 4}} d u=o\left(\Lambda^{-1 / 4}\right)
$$

as $\Lambda \rightarrow \infty$, for any constant $k$ such that $0<k<1$. Thus there is no advantage in assuming (3.6), for some $k$ arbitrarily close to 1 , in place of (2.3). In this respect the present situation is different from that of Theorem 4.1 of [5].

When proving (3.5) we shall now consider separately the contribution arising from certain neighbourhoods of $u=p\left(N^{2}\right)$ and $\lambda=N^{2}$ in the integrations with respect to $u$ and $\lambda$ respectively. To be precise, for any $\Lambda>0$, let

$$
\alpha(\Lambda)=p(\Lambda)-\left\{p^{\prime}(\Lambda)\right\}^{1 / 3}, \quad \beta(\Lambda)=p(\Lambda)+\left\{p^{\prime}(\Lambda)\right\}^{1 / 3} .
$$

For brevity we shall write $\alpha \equiv \alpha\left(N^{2}\right), \beta \equiv \beta\left(N^{2}\right)$. Further let $t_{0}$ $=N^{-1}\left\{p^{\prime}\left(N^{2}\right)\right\}^{-2 / 3}$. Then the $u$-neighbourhood to be considered separately is $(\alpha, \beta)$ and the $\lambda$-neighbourhood to be considered separately is that part of $Q_{\lambda}$ for which $|t| \leq t_{0}$, or rather $0 \leq t \leq t_{0}$, since (as usual) we shall only consider the upper half of $Q_{\lambda}$. 
It is necessary to verify that $p\left(\frac{1}{2} N^{2}\right)<\alpha<\beta<p\left(4 N^{2}\right)$. To establish the first inequality, we argue as follows. First consider the $C_{\mathrm{e}}$ case. For some $\theta$ in $\left(\frac{1}{2} N^{2}, N^{2}\right)$ and some $A>0$,

$$
\frac{p\left(N^{2}\right)-p\left(\frac{1}{2} N^{2}\right)}{\left\{p^{\prime}\left(N^{2}\right)\right\}^{1 / 3}}=\frac{\frac{1}{2} N^{2} p^{\prime}(\theta)}{\left\{p^{\prime}\left(N^{2}\right)\right\}^{1 / 3}}>A N^{2}\left\{p^{\prime}\left(N^{2}\right)\right\}^{2 / 3}>1
$$

for $N$ sufficiently large, using (3.1) of [2] and the condition $C_{\mathrm{e}}$ (iv). Hence $p\left(\frac{1}{2} N^{2}\right)<\alpha$. Next consider the $C_{1}$ case. Using (a), (b) of $\$ 2$ of [5], we have

$$
\frac{p\left(N^{2}\right)-\left\{p^{\prime}\left(N^{2}\right)\right\}^{1 / 3}}{p\left(\frac{1}{2} N^{2}\right)} \geq 2\left\{1-\frac{\left\{p^{\prime}\left(N^{2}\right)\right\}^{1 / 3}}{p\left(N^{2}\right)}\right\}>1
$$

for $N$ sufficiently large, so again $p\left(\frac{1}{2} N^{2}\right)<\alpha$. Similar proofs show that $\beta$ $<p\left(4 N^{2}\right)$.

4. Results concerning the hypothesis (2.3). In $\$ \S 4-6$ we suppose that $q(u)$ satisfies the conditions $C_{\mathrm{e}}$.

In this section we shall need to know that

$$
p^{\prime}(\lambda) /\{p(\lambda)\}^{3} \rightarrow 0, \quad \lambda\left\{p^{\prime}(\lambda)\right\}^{2 / 3} \rightarrow \infty
$$

as $|\lambda| \rightarrow \infty, \lambda \in S_{\lambda}$. The first assertion follows from Lemma 6.1 of [3], and the second from $C_{\mathrm{e}}$ (iv).

For the duration of this section define $\Lambda^{\prime}$ by $p\left(\Lambda^{\prime}\right)=\alpha(\Lambda)$. It will be shown that, as $\Lambda \rightarrow \infty$,

$$
\Lambda / \Lambda^{\prime} \rightarrow 1, \quad p(\Lambda) / p\left(\Lambda^{\prime}\right) \rightarrow 1, \quad p^{\prime}(\Lambda) / p^{\prime}\left(\Lambda^{\prime}\right) \rightarrow 1 .
$$

For some $\theta$ between $p\left(\Lambda^{\prime}\right)$ and $p(\Lambda)$,

$$
\begin{aligned}
\Lambda-\Lambda^{\prime} & =q(p(\Lambda))-q\left(p\left(\Lambda^{\prime}\right)\right)=\left\{p(\Lambda)-p\left(\Lambda^{\prime}\right)\right\} q^{\prime}(\theta) \\
& =\left\{p(\Lambda)-p\left(\Lambda^{\prime}\right)\right\} q^{\prime}(p(\phi))
\end{aligned}
$$

where $\theta=p(\phi)$, so $\phi$ is between $\Lambda^{\prime}$ and $\Lambda$. Using (3.1) of [2] it follows that, as $\Lambda \rightarrow \infty$,

$$
\Lambda-\Lambda^{\prime} \asymp\left\{p^{\prime}(\Lambda)\right\}^{-2 / 3} ;
$$

dividing by $\Lambda$, the first assertion of (4.2) follows.

Since $\left(p(\Lambda)-p\left(\Lambda^{\prime}\right)\right) / p(\Lambda)=\left\{p^{\prime}(\Lambda)\right\}^{1 / 3} / p(\Lambda) \rightarrow 0$ as $\Lambda \rightarrow \infty$, the second part of (4.2) follows. Lastly, for some $\theta$ between $\Lambda$ and $\Lambda^{\prime}$, using (3.2) of [2], it follows that

$$
\frac{p^{\prime}(\Lambda)-p^{\prime}\left(\Lambda^{\prime}\right)}{p^{\prime}(\Lambda)}=\left(\Lambda-\Lambda^{\prime}\right) \frac{p^{\prime \prime}(\theta)}{p^{\prime}(\Lambda)}=O\left(\frac{\Lambda-\Lambda^{\prime}}{\Lambda}\right) \rightarrow 0
$$


as $\Lambda \rightarrow \infty$, which gives the third part of (4.2).

Lemma 4.1. The hypothesis (2.3) is equivalent to

$$
\int_{p(\Lambda / 2)}^{a(\Lambda)} \frac{|f(u)|}{u[\Lambda-q(u)]^{1 / 4}} d u=o\left(\Lambda^{-1 / 4}\right) .
$$

Proof. First assume (2.3); then it follows that

$$
\int_{p(\Lambda / 2)}^{a(\Lambda)} \frac{|f(u)|}{u\left[\Lambda+\left\{p^{\prime}(\Lambda)\right\}^{-2 / 3}-q(u)\right]^{1 / 4}} d u=o\left(\Lambda^{-1 / 4}\right) .
$$

Now, for $p\left(\frac{1}{2} \Lambda\right) \leq u \leq \alpha(\Lambda)$, using (4.3),

$$
\begin{aligned}
\Lambda-q(u) & \geq \frac{1}{2}\{\Lambda-q(\alpha(\Lambda))\}+\frac{1}{2}\{\Lambda-q(u)\} \\
& \geq A\left\{p^{\prime}(\Lambda)\right\}^{-2 / 3}+\frac{1}{2}\{\Lambda-q(u)\} \\
& \geq B\left[\Lambda+\left\{p^{\prime}(\Lambda)\right\}^{-2 / 3}-q(u)\right]
\end{aligned}
$$

for some $A, B>0$. Hence (4.4) follows from (4.5).

Next assume (4.4); then clearly

$$
\int_{p(\Lambda / 2)}^{\alpha(\Lambda)} \frac{|f(u)|}{u\left[\Lambda+\left\{p^{\prime}(\Lambda)\right\}^{-2 / 3}-q(u)\right]^{1 / 4}} d u=o\left(\Lambda^{-1 / 4}\right) .
$$

Set $\alpha_{k}(\Lambda)=p(\Lambda)-k\left\{p^{\prime}(\Lambda)\right\}^{1 / 3}$ for fixed $k \geq 1$; then, as in $\$ 3$, it is easy to see that $\alpha_{k}(\Lambda)>p\left(\frac{1}{2} \Lambda\right)$ for $\Lambda$ sufficiently large, so using (4.3), (4.6) we have

$$
\int_{\alpha_{k}(\Lambda)}^{a(\Lambda)} \frac{|f(u)|}{u} d u=o\left(\Lambda^{-1 / 4}\left\{p^{\prime}(\Lambda)\right\}^{-1 / 6}\right) .
$$

It will be shown that $\alpha\left(\Lambda^{\prime}\right)>\alpha_{k}(\Lambda)$ for some $k$. Now, by a little manipulation,

$$
\alpha\left(\Lambda^{\prime}\right)-\alpha_{k}(\Lambda)=\left\{(k-1)\left(\frac{p^{\prime}(\Lambda)}{p^{\prime}\left(\Lambda^{\prime}\right)}\right)^{1 / 3}-1\right\}\left\{p^{\prime}\left(\Lambda^{\prime}\right)\right\}^{1 / 3} ;
$$

using (4.2) and that $p^{\prime}(\lambda)>0$ for all $\lambda$, it follows that $\alpha\left(\Lambda^{\prime}\right)>\alpha_{k}(\Lambda)$, choosing some $k>2$ and taking $\Lambda$ sufficiently large. Hence (4.7) implies that

$$
\int_{\alpha\left(\Lambda^{\prime}\right)}^{p\left(\Lambda^{\prime}\right)} \frac{|f(u)|}{u} d u=o\left(\Lambda^{\prime-1 / 4}\left\{p^{\prime}(\Lambda)\right\}^{-1 / 6}\right)
$$

as $\Lambda^{\prime} \rightarrow \infty$; thus

$$
\int_{a(\Lambda)}^{p(\Lambda)} \frac{|f(u)|}{u\left[\Lambda+\left\{p^{\prime}(\Lambda)\right\}^{-2 / 3}-q(u)\right]^{1 / 4}} d u=o\left(\Lambda^{-1 / 4}\right)
$$

which, taken with (4.6), implies (2.3). 
It is remarked that the hypothesis (2.3) implies that

$$
\int_{\beta_{k-1}(\Lambda)}^{\beta_{k}(\Lambda)} \frac{|f(u)|}{u} d u=o\left(\Lambda^{-1 / 4}\left\{p^{\prime}(\Lambda)\right\}^{-1 / 6}\right)
$$

as $\Lambda \rightarrow \infty$, for any fixed integer $k \geq 0$, where $\beta_{k}(\Lambda)=p(\Lambda)+k\left\{p^{\prime}(\Lambda)\right\}^{1 / 3}$. The case $k=0$ has already been established; induction gives the conclusion for any integer $k \geq 0$.

5. Some bounds for $\psi$. Recall that we are writing $\alpha \equiv \alpha\left(N^{2}\right), \beta \equiv \beta\left(N^{2}\right)$.

We remark that, if $0 \leq t \leq t_{0}$ and $s=N+i t$, then $\lambda \in S_{\lambda}$. For, by (4.1), $t=o(N)$, so $\nu / \mu$ is $o(1)$, and the assertion follows.

As usual, for $\lambda$ in $S_{\lambda}$, we define

$$
\zeta(u, \lambda)=\int_{0}^{p(\lambda)}\{\lambda-q(u)\}^{1 / 2} d u .
$$

Lemma 5.1. Let $s=N+i t, u \in(\alpha, \beta)$ and $N \rightarrow \infty$; then

$$
|\zeta(u, \lambda)|=O\left\{|\lambda-q(u)|^{3 / 2} p^{\prime}\left(N^{2}\right)\right\} .
$$

If, in addition, $0 \leq t \leq t_{0}$ then

$$
|\lambda-q(u)|=O\left\{\left[p^{\prime}\left(N^{2}\right)\right]^{-2 / 3}\right\} .
$$

Proof. From the definition of $\zeta$ we have

$$
\zeta(u, \lambda)=\int_{\lambda}^{q(u)}(\lambda-\tau)^{1 / 2} p^{\prime}(\tau) d \tau=O\left(|\lambda-q(u)|^{3 / 2} \sup \left|p^{\prime}(\tau)\right|\right),
$$

the supremum being taken over the path of integration; if we take this to be a straight line, and use (3.1) of [2], then (5.1) follows.

Now suppose that $0 \leq t \leq t_{0}$. Then

$$
|\lambda-q(u)|=\left\{\left|N^{2}-t^{2}-q(u)\right|^{2}+4 N^{2} t^{2}\right\}^{1 / 2} \leq\left|N^{2}-q(u)\right|+t^{2}+2 N t,
$$

which is easily seen to be $O\left(\left\{p^{\prime}\left(N^{2}\right)\right\}^{-2 / 3}\right)$.

The results (5.1), (5.2) together imply that $\zeta(u, \lambda)$ is bounded, as $N \rightarrow \infty$, for $u \in(\alpha, \beta)$ and $0 \leq t \leq t_{0}$.

If $z(=x+i y) \in C$ and $a \in R$ it is trivial that $\left|z^{2}-a^{2}\right| \geq\left|x^{2}-a^{2}\right|$. If $\lambda \in Q_{\lambda}$,

$$
|\lambda-q(u)|>\left|N^{2}-q(u)\right| \text {. }
$$

For, if $\lambda=(N+i t)^{2}$ and $0 \leq t \leq N$, (5.3) follows immediately; if $\lambda=$ $(\sigma+i N)^{2}$ and $0 \leq \sigma \leq N$, then

$$
\left|(\sigma+i N)^{2}-q(u)\right| \geq\left|\sigma^{2}-\left(N^{2}+q(u)\right)\right| \geq q(u) \geq\left|N^{2}-q(u)\right| .
$$


Lemma 5.2. Suppose that $q(u)$ satisfies the conditions $C_{\mathrm{e}}$. Let $\lambda \in Q_{\lambda}$, $s$ $=N+i t, 0 \leq t \leq t_{0}$, and $u \in(\alpha, \beta)$. Then

$$
\psi(u, \lambda)=O\left[N^{-1 / 2}\left\{p^{\prime}\left(N^{2}\right)\right\}^{1 / 6} \exp \left\{-t p\left(N^{2}\right)\right\}\right] .
$$

Proof. We proceed as in $\S 8$ of [3] except that now, since $|\zeta(u, \lambda)|=O(1)$, we may use the bound $H_{1 / 3}{ }^{(1)}(\zeta)=O\left(|\zeta|^{-1 / 3}\right)$ instead of (8.1) of [3]. Thus

$$
\psi(u, \lambda)=O\left\{\frac{|\zeta(u, \lambda)|^{1 / 6} \exp \{\operatorname{im} \zeta(0, \lambda)\}}{\lambda^{1 / 4}|\lambda-q(u)|^{1 / 4}}\right\}
$$

which gives (5.4) on using (5.1) and Lemma 3.1 of [3].

Lemma 5.3. Suppose that $q(u)$ satisfies the conditions $C_{\mathrm{e}}$. Let $\lambda \in Q_{\lambda}$ and $u \in\left(\beta, p\left(4 N^{2}\right)\right)$; then

$$
\psi(u, \lambda)=O\left\{\frac{\exp -\left[t p\left(N^{2}\right)+A p^{\prime}\left(N^{2}\right)\left\{q(u)-N^{2}\right\}^{3 / 2}\right]}{|s|^{1 / 2}\left|s^{2}-q(u)\right|^{1 / 4}}\right\}
$$

where $A$ is a positive constant.

Proof. Let $\lambda-q(u)=r e^{i \theta}$; then as in $\S 9$ of [3], if $u>p\left(N^{2}\right) \geq p(\mu)$, since $N^{2} \geq \mu$, we have $\frac{1}{2} \pi \leq \theta \leq \pi$. Hence, for $\lambda \in Q_{\lambda}$, using (5.3)

$$
\operatorname{im}\{\lambda-q(u)\}^{1 / 2}=|\lambda-q(u)|^{1 / 2} \sin \frac{1}{2} \theta \geq\left\{q(u)-N^{2}\right\}^{1 / 2} / \sqrt{ } 2 .
$$

Therefore

$$
\begin{aligned}
\operatorname{im} \xi(u) & =\int_{0}^{p\left(N^{2}\right)} \operatorname{im}\{\lambda-q(v)\}^{1 / 2} d v+\int_{p\left(N^{2}\right)}^{u} \operatorname{im}\{\lambda-q(v)\}^{1 / 2} d v \\
& \geq t p\left(N^{2}\right)+\frac{1}{\sqrt{ } 2} \int_{p\left(N^{2}\right)}^{u}\left\{q(v)-N^{2}\right\}^{1 / 2} d v \\
& \geq t p\left(N^{2}\right)+\frac{3}{2} A p^{\prime}\left(N^{2}\right) \int_{p\left(N^{2}\right)}^{u}\left\{q(v)-N^{2}\right\}^{1 / 2} q^{\prime}(v) d v
\end{aligned}
$$

since $v \in\left(p\left(N^{2}\right), p\left(4 N^{2}\right)\right)$, and using (3.1) of [2], where $A>0$. Thus

$$
\operatorname{im} \xi(u) \geq t p\left(N^{2}\right)+A p^{\prime}\left(N^{2}\right)\left\{q(u)-N^{2}\right\}^{3 / 2},
$$

and (5.5) follows.

6. Proof of the main result. We are now in a position to establish (3.5). It was explained in $\$ 3$ that we shall consider separately the part of (3.5) arising from $\alpha \leq u \leq \beta$, in turn dealing separately with $0 \leq t \leq t_{0}$ and $t>t_{0}$.

Using Lemma 5.2 above and Lemma 4.1 of [4] it follows that the contribution of $\alpha \leq u \leq \beta, s=N+i t, 0 \leq t \leq t_{0}$ to the left side of (3.5) is of magnitude 


$$
\begin{gathered}
o\left\{N^{1 / 2}\left\{p^{\prime}\left(N^{2}\right)\right\}^{1 / 6} \int_{0}^{t_{0}}\left[\int_{\alpha}^{\beta} \exp \left\{t\left(x-p\left(N^{2}\right)\right)\right\}|f(u)| d u\right] d t\right\} \\
=o\left\{\frac{N^{1 / 2}\left\{p^{\prime}\left(N^{2}\right)\right\}^{1 / 6}}{p\left(N^{2}\right)} \int_{\alpha}^{\beta}|f(u)| d u\right\}=o(1),
\end{gathered}
$$

using Fubini's theorem and (4.8).

Next we consider the contribution arising from $\alpha \leq u \leq \beta, t>t_{0}$.

First suppose that $\alpha \leq u \leq p\left(N^{2}\right)$. Set $v^{2}=q(u)$; then

$$
\begin{aligned}
\left|(N+i t)^{2}-q(u)\right| & =\left\{(N+v)^{2}+t^{2}\right\}^{1 / 2}\left\{(N-v)^{2}+t^{2}\right\}^{1 / 2} \\
& \geq \frac{1}{2}(N+v+t)(N-v+t) .
\end{aligned}
$$

Since $t>t_{0}=N^{-1}\left\{p^{\prime}\left(N^{2}\right)\right\}^{-2 / 3}$ it follows that

$$
\left|(N+i t)^{2}-q(u)\right| \geq \frac{1}{2}\left[N^{2}+\left\{p^{\prime}\left(N^{2}\right)\right\}^{-2 / 3}-q(u)\right] .
$$

Hence the contribution of $\alpha \leq u \leq p\left(N^{2}\right), s=N+i t, t_{0} \leq t \leq N$ to the left side of (3.5) is

$$
O\left\{N^{1 / 2} \int_{0}^{N}\left[\int_{\alpha}^{p\left(N^{2}\right)} \frac{e^{t(x-u)}|f(u)|}{\left[N^{2}+\left\{p^{\prime}\left(N^{2}\right)\right\}^{-2 / 3}-q(u)\right]^{1 / 4}} d u\right] d t\right\}
$$

which is $o(1)$, using Fubini's theorem again and (2.3).

Clearly

$$
\left|(\sigma+i N)^{2}-v^{2}\right|=|\sigma+i N+v||\sigma+i N-v| \geq N^{2},
$$

so the contribution of $\alpha \leq u \leq p\left(N^{2}\right), s=\sigma+i N, 0 \leq \sigma \leq N$ is

$$
o\left\{\int_{0}^{N} d \sigma \int_{\alpha}^{p\left(N^{2}\right)} e^{N(x-u)}|f(u)| d u\right\}=o\left\{N \int_{\alpha}^{\alpha\left(N^{2}\right)} e^{-N u / 2}|f(u)| d u\right\}
$$

which is easily seen to be $o(1)$.

Next suppose that $p\left(N^{2}\right) \leq u \leq \beta$ and $t>t_{0}$. Then, as for (6.1), we have

$$
\left|(N+i t)^{2}-q(u)\right| \geq \frac{1}{2}\left[q(u)+\left\{p^{\prime}\left(N^{2}\right)\right\}^{-2 / 3}-N^{2}\right] .
$$

Therefore the contribution of $p\left(N^{2}\right) \leq u \leq \beta, s=N+i t, t_{0}<t \leq N$ is

$$
\begin{aligned}
o\left\{N^{1 / 2} \int_{0}^{N}\left[\int_{\left.p^{(N 2}\right)}^{\beta} \frac{e^{t(x-u)}|f(u)|}{\left[q(u)+\left\{p^{\prime}\left(N^{2}\right)\right\}^{-2 / 3}-N^{2}\right]^{1 / 4}} d u\right] d t\right\} \\
\quad=O\left\{N^{1 / 2}\left\{p^{\prime}\left(N^{2}\right)\right\}^{1 / 6} \int_{0}^{N}\left[\int_{p\left(N^{2}\right)}^{\beta} e^{f(x-u)}|f(u)| d u\right] d t\right\}
\end{aligned}
$$

which is seen to be $o(1)$, using Fubini's theorem and (4.8). 
Thus we have shown so far that all the parts of the contribution arising from $\alpha \leq u \leq \beta$ are $o(1)$ as $N \rightarrow \infty$.

Using (5.3), the contribution arising from $p\left(\frac{1}{2} N^{2}\right) \leq u \leq \alpha, s=N+i$, $0 \leq t \leq N$ is

$$
O\left\{N^{1 / 2} \int_{0}^{N}\left[\int_{p\left(N^{2} / 2\right)}^{\alpha} \frac{e^{t(x-u)}|f(u)|}{\left\{N^{2}-q(u)\right\}^{1 / 4}} d u\right] d t\right\}
$$

which is $o(1)$ by Lemma 4.1. The contribution arising from $s=\sigma+i N$ is clearly of no larger magnitude, so also is $o(1)$.

By Lemma 5.3 and (5.3), the contribution arising from $\beta \leq u \leq p\left(4 N^{2}\right)$, $s=N+i t, 0 \leq t \leq N$ is

$$
O\left\{N^{1 / 2} \int_{0}^{N}\left[\int_{\beta}^{p\left(4 N^{2}\right)} \frac{\exp \left[t x-t p\left(N^{2}\right)-A p^{\prime}\left(N^{2}\right)\left\{q(u)-N^{2}\right\}^{3 / 2}\right]}{\left\{q(u)-N^{2}\right\}^{1 / 4}}|f(u)| d u\right] d y\right\}
$$

which is

$$
o\left\{\frac{N^{1 / 2}}{p\left(N^{2}\right)} \int_{\beta}^{p\left(4 N^{2}\right)} \frac{\exp \left[-A p^{\prime}\left(N^{2}\right)\left\{q(u)-N^{2}\right\}^{3 / 2}\right]}{\left\{q(u)-N^{2}\right\}^{1 / 4}}|f(u)| d u\right\} .
$$

To deal with this term we imitate the argument employed by Muckenhoupt (see $\$ 3$ of [1]).

Temporarily set $a=p\left(N^{2}\right), b=\left\{p^{\prime}\left(N^{2}\right)\right\}^{1 / 3}$. Then it is easy to see that for a given $N$ there exists $j$ such that

$$
\left[a+b, p\left(4 N^{2}\right)\right] \subseteq \bigcup_{k=1}^{j}[a+k b, a+(k+1) b] \subseteq\left[a+b, p\left(8 N^{2}\right)\right] .
$$

Now, for $k=1,2, \ldots$, for some $\theta$ in $(a, a+k b)$, and hence in $\left(p\left(N^{2}\right), p\left(8 N^{2}\right)\right)$,

$$
q(a+k b)-N^{2}=q(a+k b)-q(a)=k b q^{\prime}(\theta) .
$$

so $\left\{p^{\prime}\left(N^{2}\right)\right\}^{2 / 3}\left\{q(a+k b)-N^{2}\right\} \rightarrow k$ as $N \rightarrow \infty$. Hence (6.2) is

$$
\begin{gathered}
o\left\{\frac{N^{1 / 2}\left\{p^{\prime}\left(N^{2}\right)\right\}^{1 / 6}}{p\left(N^{2}\right)} \sum_{k=1}^{\infty} \int_{a+k b}^{a+(k+1) b} \frac{\exp \left(-A k^{3 / 2}\right)}{k^{1 / 4}}|f(u)| d u\right\} \\
=o\left\{\frac{1}{p\left(N^{2}\right)} \sum_{k=1}^{\infty} \frac{\{a+(k+1) b\} \exp \left(-A k^{3 / 2}\right)}{k^{1 / 4}}\right\}
\end{gathered}
$$

using (4.8); thus (6.2) is $o(1)$. This completes the proof of Theorem 2.1 in the $C_{e}$ case.

7. The $C_{1}$ case. We outline the steps in the arguments of $\$ \$ 4,5,6$ which have to be changed when $q(u)$ satisfies the conditions $C_{1}$ instead of $C_{\mathrm{e}}$. The statements of the results of $\S 4$ are unchanged, but some modifications have to be made to those of Lemmas 5.2, 5.3. 
The first part of (4.1) is (2.1) of [5], and the second part follows from the first part of (2.2) of [5]. To establish the first two parts of (4.2) the arguments are changed only to the extent of using (2.3) of [5] in place of (3.1), (3.2) of [2]. For the third part of (4.2) we have, for some $\theta$ between $\Lambda$ and $\Lambda^{\prime}$,

$$
\frac{p^{\prime}(\Lambda)-p^{\prime}\left(\Lambda^{\prime}\right)}{p^{\prime}(\Lambda)}=\left(\Lambda-\Lambda^{\prime}\right) \frac{p^{\prime \prime}(\theta)}{p^{\prime}(\Lambda)}=O\left(\left(\Lambda-\Lambda^{\prime}\right) \frac{p^{\prime}(\Lambda)}{p(\Lambda)}\right)
$$

which is $o(1)$ as $\Lambda \rightarrow \infty$, by (4.3) and (4.1).

In $\$ 5$ first note that if $0 \leq t \leq t_{0}$, for some $\theta$ in $\left(N^{2}-t^{2}, N^{2}\right), p\left(N^{2}\right)$ $-p\left(N^{2}-t^{2}\right)=t^{2} p^{\prime}(\theta) \leq t^{2} p^{\prime}\left(N^{2}\right)$ so $p\left(N^{2}\right) \asymp p\left(N^{2}-t^{2}\right)$. Hence, for $s=N$ $+i t\left(\right.$ so $\left.\mu=N^{2}-t^{2}\right)$ and $0 \leq t \leq t_{0}$,

$$
\nu p^{\prime}(\mu) / p(\mu) \leq A\left\{p^{\prime}\left(N^{2}\right)\right\}^{1 / 3} / p\left(N^{2}\right)=o(1)
$$

and thus $\lambda \in S_{\lambda}$.

In the first paragraph of the proof of Lemma 5.1 we use $C_{1}(v)$ and (2.3) of [5] in place of (3.1) of [2]. No other modification is required.

Let $g(\mu)=\left\{\mu p(\mu) p^{\prime}(\mu)\right\}^{1 / 2}$; the function $g$ first arose in $\$ 5$ of [5]. In place of Lemmas 5.2, 5.3 we now have the following.

Lemma 7.1. Suppose that $q(u)$ satisfies the conditions $C_{1}$. Let $\lambda \in Q_{\lambda}, s=N+i$. $0 \leq t \leq t_{0}$ and $u \in(\alpha, \beta)$; then

$$
\psi(u, \lambda)=O\left[N^{-1 / 2}\left\{p^{\prime}\left(N^{2}\right)\right\}^{1 / 6} \exp \left\{-\operatorname{tg}\left(N^{2}\right)\right\}\right] .
$$

Lemma 7.2. Suppose that $q(u)$ satisfies the conditions $C_{1}$. Let $\lambda \in Q_{\lambda}$ and $u \in\left(\beta, p\left(4 N^{2}\right)\right)$; then

$$
\psi(u, \lambda)=O\left\{\frac{\exp -\left[\operatorname{tg}\left(N^{2}\right)+A p^{\prime}\left(N^{2}\right)\left\{q(u)-N^{2}\right\}^{3 / 2}\right]}{|s|^{1 / 2}\left|s^{2}-q(u)\right|^{1 / 4}}\right\}
$$

where $A$ is a positive constant.

These results are easily established by modifying the proofs of Lemmas 5.2, 53 according to the results of $\$ 5$ of [5].

The changes in Lemmas 5.2, 5.3 necessitate slight modifications to the arguments of $\S 6$; these are all straightforward.

8. Proof of Theorem 2.3. This result is established by using Hölder's inequality. Let $s$ be defined by $r^{-1}+s^{-1}=1$.

First consider the $C_{\mathrm{e}}$ case. Then, as shown in $\S 1$ of [4], for any fixed $a>0$, $p(a \Lambda) \asymp p(\Lambda)$ as $\Lambda \rightarrow \infty$; thus 


$$
\begin{aligned}
\int_{p(\Lambda / 2)}^{p(\Lambda)} & \frac{|f(u)|}{u\left[\Lambda+\left\{p^{\prime}(\Lambda)\right\}^{-2 / 3}-q(u)\right]^{1 / 4}} d u \\
& \leq \frac{A}{p(\Lambda)} \int_{p(\Lambda / 2)}^{p(\Lambda)} \frac{|f(u)|}{\left[\Lambda+\left\{p^{\prime}(\Lambda)\right\}^{-2 / 3}-q(u)\right]^{1 / 4}} d u \\
& \leq \frac{A}{p(\Lambda)}\left(\int|f(u)|^{r} d u\right)^{1 / r}\left(\int \frac{d u}{\left[\Lambda+\left\{p^{\prime}(\Lambda)\right\}^{-2 / 3}-q(u)\right]^{s / 4}}\right)^{1 / s} \\
& <\frac{A\left\{p^{\prime}(\Lambda)\right\}^{1 / s}}{p(\Lambda)}\left(\int|f(u)|^{r} d u\right)^{1 / r}\left(\int \frac{q^{\prime}(u)}{\left[\Lambda+\left\{p^{\prime}(\Lambda)\right\}^{-2 / 3}-q(u)\right]^{s / 4}} d u\right)^{1 / s}
\end{aligned}
$$

by (3.1) of [2]. Now using (2.4) and that $p(2 \Lambda) \asymp p(\Lambda)$ we can deduce (2.3).

For the $c_{1}$ case we can no longer use that $p(a \Lambda) \asymp p(\Lambda)$ or (3.1) of [2]; instead we have to make do with the monotonicity of $p(\Lambda), p^{\prime}(\Lambda)$. With the aid of these (2.3) is then deduced in a similar way from (2.4).

\section{REFERENCES}

1. B. Muckenhoupt, Equiconvergence and almost everywhere convergence of Hermite and Laguerre series, SIAM J. Math. Anal. 1 (1970), 295-321. MR 42 \#4948.

2. C. G. C. Pitts, Asymptotic approximations to solutions of a second-order differential equation, Quart. J. Math. Oxford Ser. (2) 17 (1966), 307-320. MR 34 \#6254.

3._- Simplified asymptotic approximations to solutions of a second-order differential equation, Quart. J. Math. Oxford Ser. (2) 21 (1970), 223-242.

4.__, An equiconvergence result of eigenfunction expansions for a pasitive increasing potential function, Quart. J. Math. Oxford Ser. (2) 21 (1970), 357-369. MR 42 \#486.

5.—, On eigenfunction expansions for a positive potential function increasing slowly to infinity, J. Differential Equations 13 (1973), 358-373.

6. G. Szegö, Orthogonal polynomials, rev. ed., Amer. Math. Soc. Colloq. Publ., vol. 23, Amer. Math. Soc., Providence, R.I., 1959. MR 21 \# 5029.

School of Mathematics and Physics, University of East Anglia, Norwich NOR 88C, ENGLAND 